\title{
Determination of Magnetospheric Wave Polarization Using Ground Based Sun-Rise Geomagnetic Observations
}

\author{
L. ALPEROVICH ${ }^{1}$, B. FIDEL ${ }^{1}$, and O. SAKA ${ }^{2}$ \\ ${ }^{1}$ Department of Geophysics and Planet. Science, Tel-Aviv University, Ramat-Aviv 69978, Israel \\ ${ }^{2}$ Department of Earth and Planet. Science, Kyushu University, Fukuoka 812, Japan
}

(Received December 15, 1994; Revised October 9, 1995; Accepted November 13, 1995)

\begin{abstract}
A new experimental method is suggested to recognize a type of the incident magnetospheric wave following by the time and frequency dependency of the azimuth angle of geomagnetic pulsations close to the terminator. The main idea is based on the fundamental differences between the ionospheric transformation of the Alfvén and magnetosonic waves. The Alfvén waves are associated with some kind of field aligned currents which cannot penetrate into the atmosphere and spread over the high conductive ionosphere. Magnetic effect on the ground is defined mainly by the ionospheric currents caused by the Alfvén wave. In contrast, in the case of magnetosonic wave, the ground current system provides the main contribution to the total current and going from the daylight ionosphere to the night one does not markedly affects on the total current. Equatorial observations revealed the horizontal orientation angle in different frequency ranges. This is inclined to think that in the cases have been under the consideration the oscillations within the range $50 \sim 500 \mathrm{~s}$ are generated by the Alfvén waves.
\end{abstract}

\section{Introduction}

At present there are two alternative methods of explanation of a ground pulsation structure. One of them is based on the assumption that hydromagnetic waves originate in the magnetosphere and come to the ionosphere in the Alfvén mode (see, for example, Chen and Hasegawa, 1974; Hughes and Southwood, 1976). Ariother way of looking at the nature of geomagnetic pulsations is the idea dealing with the magnetosonic waves falling from the magnetosphere onto the ionosphere. Yumoto and Saito (1983) and Yumoto (1986) suggested that there is definite sort of low-latitude variations which originate due to the magnetosonic (poloidal) resonance in the plasmasphere. Contemporary theory of generation of such oscillations in the region of resonant magnetic shells was developed in the paper of $\mathrm{Zhu}$ and Kivelson (1988). Moreover, reality of these oscillations was proved experimentally by comparing satellite data on magnetic pulsations with fluctuations of middle and high energetic protons (Zhu and Kivelson, 1994).

The low latitude observations in the special region like terminator or geomagnetic equator can give additional facts concerning a nature of waves incoming from the magnetosphere to the ionosphere.

Here we present results of numerical simulations of Alfvén and fast magnetosonic waves propagating through the inhomogeneous ionosphere, as well as a method of separating incoming magnetospheric waves according to their polarization, Alfvén or magnetosonic mode.

\section{Homogeneous Ionosphere}

Alperovich and Fedorov (1984) and Alperovich et al. (1991) considered a propagation of arbitrary polarized magnetohydrodynamic waves in the plane-layered system. Within the limits of ideal magnetohydrodynamics, the magnetosphere is modeled by an anisotropic half-space with infinite dielectric permeability along the main magnetic field and finite cross permeability $\varepsilon_{m} \sim V_{\mathrm{a}}{ }^{-2}$, where $V_{\mathrm{a}}$ is the Alfvén velocity. The ionosphere is chosen as a thin anisotropic sheet with tensor integral conductivity $\Sigma_{i k}$. The atmosphere and the Earth in the model have scalar conductivities $\sigma_{a}$ and $\sigma_{g}$, respectively. 
The deduced analytical expression for matrixes of reflection and transformation enable to examine a behavior of the electromagnetic field caused by the hydromagnetic wave above and below the ionosphere as a function of ionospheric conductivity. We put the Alfvén velocity $V_{\mathrm{a}}=10^{6} \mathrm{~m} / \mathrm{s}$ and the Earth is a half space with constant conductivity $\sigma_{g}=10^{-1}(\mathrm{Ohm} \cdot \mathrm{m})^{-1}$. The inclination of the geomagnetic field is $I=20^{\circ}$. The initial wave was assumed to be a monochromatic wave with period $T=100 \mathrm{~s}$, meridional and azimuth wave numbers $k_{x}=10^{-5} \mathrm{~m}^{-1}$ and $k_{y}=10^{-6} \mathrm{~m}^{-1}$ respectively. Amplitude of the horizontal magnetic component of the incident wave was chosen to be equal to $1 \mathrm{nT}$.

The most sensitive to variations of the ionospheric conductivity in the case of the initial Alfven wave is its electric field in the ionosphere (see solid lines in Fig. 1). Magnitude of the total electric field is inversely proportional to integral Pedersen conductivity $\Sigma_{p}$. The maximum ionospheric electric field induced by incoming Alfvén waves appears on the night side of the ionosphere because of conservation of its field-aligned current.

Concerning the influence of the ionosphere on the magnetosonic electric field (dashed lines in Fig. 1), one can see that the field rotates on $30^{\circ}$ approximately, when going from day to night conditions, and it has, in fact, the azimuth direction in the night. Nevertheless, the magnetic component under the ionosphere and on the Earth's surface does not follow the rotation of the ionospheric electric fields and currents (see Fig. 2, dashed lines).

We can explain it thus. The skin-layer depth in the conductive Earth $\delta_{g} \propto \sqrt{T / \sigma_{g}}$. For typical values of $\sigma_{g} \sim 0.1 \mathrm{Ohm}^{-1} \cdot \mathrm{m}^{-1}$ and $T=100 \mathrm{~s}$, we have $\delta_{g} \sim 2 \cdot 10^{4} \mathrm{~m}$. Total current $J_{g}$ in the Earth is $J_{g} \sim E_{g} \cdot \delta_{g} \cdot \sigma_{g}$. The electric field in the Earth $E_{\mathrm{g}}$ induced by the fast magnetosonic wave with amplitude of incident wave $B^{i}=1 \mathrm{nT}$ and period $100 \mathrm{~s}$ is $E_{g} \sim 2 \cdot 10^{-3} \mathrm{mV} / \mathrm{m}$. Hence $J_{g} \sim 4 \mathrm{~mA} / \mathrm{m}$. An electric field in the ionosphere is $E_{i} \sim 6 \cdot 10^{-3} \mathrm{mV} / \mathrm{m}$ (see Fig. 1), and integral conductivity of the ionosphere is $10 \mathrm{Ohm}^{-1}$. Therefore, the total ionospheric current is $J_{i} \sim 6 \cdot 10^{-2} \mathrm{~mA} / \mathrm{m}$, and, it is essentially less then the ground current. Transition from the day to the night conditions does not influence on the magnitude of the magnetic component in the atmosphere and on the Earth's surface. Thus, the homogeneous ionosphere is transparent for the FMSwaves. Magnetic field on the ground and under the ionosphere is determined mainly by the currents inside the Earth.

In the Alfvén wave the magnetic field under the ionosphere appears due to the ionospheric currents which are the source of the magnetotelluric field. Magnetic field in the ionosphere in this case is

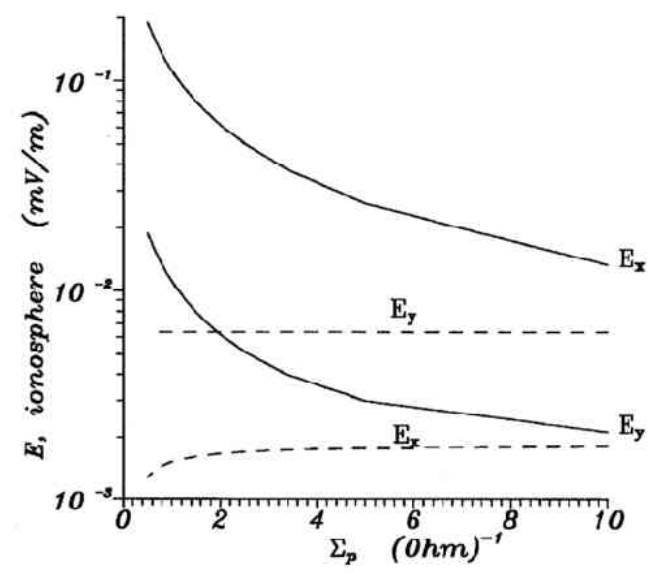

Fig. 1. Dependence of the $E_{x}$ and $E_{y}$ components of the electric field in the ionosphere on its integral Pedersen conductivity $\Sigma_{p}$. The magnetosphere is assumed to be a half-space with Alfvén velocity $V_{\mathrm{a}}=10^{6} \mathrm{~m} / \mathrm{s}$. The Earth is assumed to be a half-space with conductivity $\sigma_{g}=0.1(\mathrm{Ohm} \cdot \mathrm{m})^{-1}$. The thickness of the atmosphere $h=10^{5} \mathrm{~m}$. The magnetic component of the incident wave was chosen to be 1 . Solid lines correspond to the Alfvén wave. Dashed lines correspond to the magnetosonic wave. Here and below $x$ is directed to the South and $y$ to the East. 


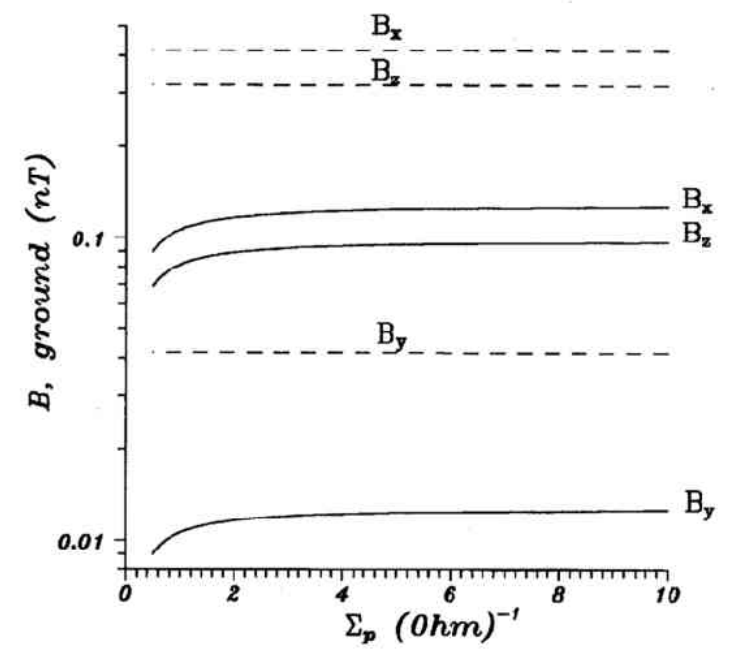

Fig. 2. $B_{x}$ and $B_{y}$ components of the Alfvén (solid line) and magnetosonic waves (dashed line) on the ground as functions of $\Sigma_{P}$.

approximately more by factor 2 than that on the ground. Let us note that despite of a significant difference in the mechanisms of ionospheric transformation of the Alfvén and magnetosonic waves, it is impossible to explain the $\sim 45^{\circ}$ rotation of the horizontal magnetic vector on the ground within the framework of the model of the homogeneous ionosphere.

\section{Inhomogeneous Ionosphere}

We studied a current distribution caused by the hydromagnetic waves in the ionosphere in which the electron density and the conductivity are defined by the solar zenith angle. A significant difference of the conductivities on the sunlight and night sides also has taken account.

Glassmeier and Heppner (1992) separated a special type of the high-latitude long-period pulsations, associated with a couple of field-aligned currents. Their magnetic effect on the ground may be expressed in terms of the twin vortex equivalent current system that moves along the Earth's surface with a speed of $\sim 2-5 \cdot 10^{3} \mathrm{~m} / \mathrm{s}$. We considered a case when these field-aligned currents are localized in the high-latitude region near the morning terminator. Such a model corresponds to the source of the Alfvén waves, generated in the flanks of the magnetosphere.

The main idea of our numerical simulations is following. The total electric current $J(r)$ of the Alfvén wave must be exactly equal to the ionospheric current. Other words, if we know the spatial distribution of the field aligned currents and coordinate dependencies of the integral conductivities $\Sigma_{i k}(\boldsymbol{r})$, it is possible to find the distribution of potential of the electric field solving the equation

$$
\operatorname{Div} \perp\left(\Sigma_{i k} \nabla \phi\right)=J(\boldsymbol{r})
$$

Here Div $\perp$ is the two-dimensional divergency. With knowledge of the $\phi$, it is possibly to construct a system of the electric fields $-\nabla \phi$, currents $-\Sigma_{i k} \nabla \phi$ and magnetic fields induced by the Alfvén wave.

One can see, that the intensity of the electric currents (see vectors Fig. 3 ) at the equator may reach $\sim 25 \%$ from their maximum values near the source. The magnetic vectors are directed perpendicularly to the terminator line. The latter leads to its significant inclination with respect to the meridional plane. Thus, Alfvén oscillations may explain the experimentally observed magnetic vector rotation at the equator(Saka et al., 1982). 


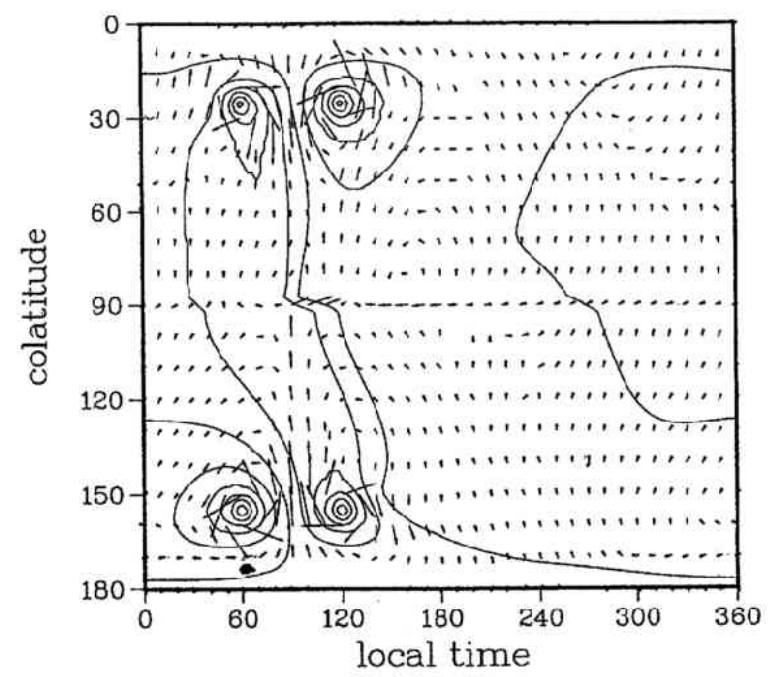

Fig. 3. Two-vortex ionospheric equivalent current system arising due to the reconnection of the interplanetary with geomagnetic field. Two field-aligned currents of the Alfvén wave inflow in each hemisphere. Equinox.

\section{Discussion}

There are some fundamental differences between the mechanism of the ionospheric transformation of magnetohydrodynamic waves of the Alfvén and magnetosonic mode. The Alfvén waves are associated with some kind of field-aligned currents. Roughly, the current flow for such inserting is defined by the total impedance. In so doing, the atmosphere with almost infinite resistance provides the main contribution. The field-aligned currents cannot penetrate into the atmosphere and spread over the high conductive ionosphere. Magnetic effect on the ground is defined mainly by the ionospheric currents caused by the Alfvén wave. The electric component of the Alfvén waves will be more in the night in compare with the day value. Space and time distortions of the magnetic field on the ground induced by the anomalies of the ionospheric conductivities may not depend on the frequency because the skin layer of electromagnetic waves is more than the thickness of the ionospheric $E$-layer for the whole pulsation range.

In contrast, in the case of magnetosonic wave, we deal with parallel connection in the magnetosphereEarth system. A current in each branch is equal to electric field of the wave divided by the apparent resistance of the branch and the total current is the sum of the partial branch current. Integral conductivity of the ground skin layer is more even than the integral conductivity of the daylight ionosphere. Therefore the ground system provides the main contribution to the total current and going from the daylight ionosphere to the night one does not markedly affects on the total current.

We can propose a scheme for definition of polarization of initial magnetospheric waves based on the ground observations. First of all, it is ought to filter observed pulsations into different frequency ranges and follow the course of the orientation angle as a function of time and on every frequency. If the angle does not change on some frequency it is evidence for the magnetosonic nature of the wave. Conversely, if the angle fluctuates either at the sunset or sunrise time, it means that the Alfvén wave incidents onto the ionosphere.

We applied this procedure to the geomagnetic pulsations observed at the equatorial observatory Huankayo. Figure 4 demonstrates the orientation angle dependence of pulsation horizontal vector on the local time LT for three ranges: $<50,50 \sim 150,150 \sim 500 \mathrm{~s}$. It is clear that the sunrise effect takes place within all ranges, and the magnetic vector changes its direction up to $45^{\circ}$ near the terminator. One can see that there is sunrise anomaly of the orientation angle in each range. It leads to the doubt on the poloidal nature 


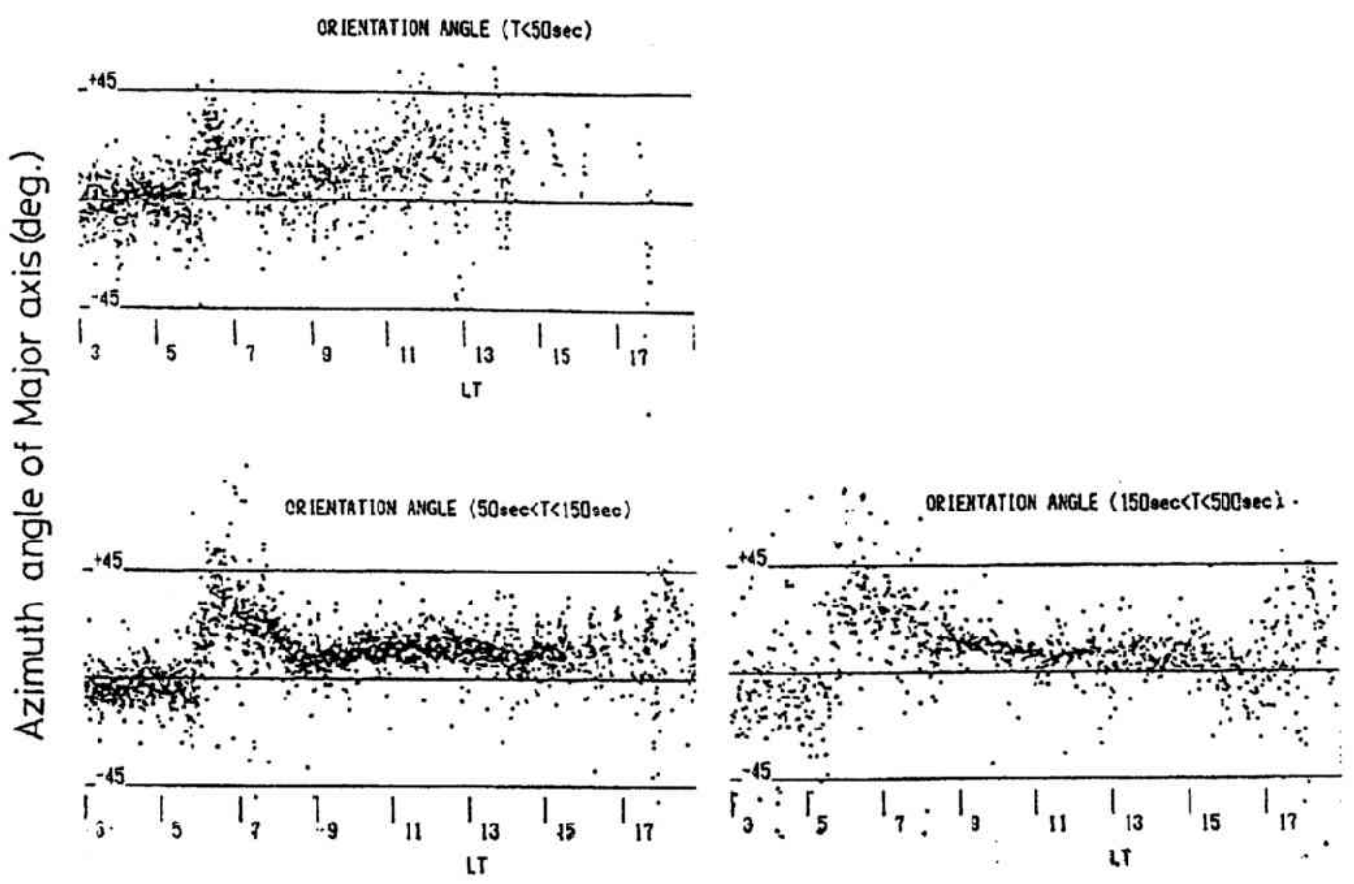

Fig. 4. Dependence of the orientation angle (in degrees) of the horizontal magnetic field on local time for pulsations within three different ranges $T<50 \mathrm{~s} ; 50 \mathrm{~s}<T<150 \mathrm{~s}, 150 \mathrm{~s}<T<500 \mathrm{~s}$.. The equatorial Observatory Huancayo, 04/25/1986.

of these pulsations and one is inclined to think that in the cases have been under the consideration the oscillations within the range $50 \sim 500 \mathrm{~s}$ are generated by the Alfvén waves.

For the further increasing of the reliability of the initial wave type determination it is desirable to have at least a couple of observatories which are placed at the long distance along a parallel. One of them must be located on the cross-section with a small resistance, and the other one on the cross-section with a large resistance. If we observe the anomaly in both observatories it will indicate to the Alfvén wave nature. If the low latitude pulsations arise as a result of poloidal oscillations of the plasmasphere, anomalies of the ionospheric conductivity will not influence the polarization features of pulsations in this place. Anomalies in this case can occur only in observatory located in a high-resistant section.

\section{REFERENCES}

Alperovich, L. S. and E. N. Fedorov, The propagation of hydromagnetic waves through the ionospheric plasma and spatial characteristics of the geomagnetic variations, Geomagn. Aeron., 24, 539-534, 1984.

Alperovich, L. S., E. N. Fedorov, and T. N. Osmakova, On the features of magnetotelluric field nearest to the resonance shell, Physics of the Solid Earth, 7, 60-71, 1991.

Chen, L. and A. Hasegawa, A theory of long-period magnetic pulsations 1., Steady state excitation of field line resonance, J. Geophys. Res., 79, 1024-1032, 1974.

Glassmeir, K. H. and C. Heppner, Traveling magnetospheric convection twin vortices: another case study, global characteristics, and a model, J. Geophys. Res., 97, 3977-3995, 1992.

Hughes, W. J. and D. J. Southwood, The screening of micropulsation signals by the atmosphere and ionosphere, J. Geophys. Res., 81, 3234-3240, 1976.

Saka, O., M. Itonaga, and T. Kitamura, Ionospheric control of polarization of low-latitude geomagnetic micropulsations at sunrise, J. Atmos. Terr. Phys., 44, 703-712, 1982.

Yumoto, K., Generation and propagation mechanisms of low-latitude magnetic pulsations-A review, J. Geophys., 60, 79-105, 1986. 
Yumoto, K. and T. Saito, Relation of compressional HM waves at GOES 2 to low-latitude Pc3 magnetic pulsations, J. Geophys. Res., 88, 10041-10052, 1983.

Zhu, X. and M. Kivelson, Analytic formulation and quantitative solutions of the coupled ULF wave problem, J. Geophys. Res., 93, 8602-8612, 1988.

Zhu, X. and M. Kivelson, Compressional ULF waves in the outer magnetosphere: 2 . A case study of Pc5 type wave activity, $J$. Geophys. Res., 99, 241-252, 1994. 